gen in Komorbidität mit PTSD führen nämlich oft zur Chronifizierung und Therapieresistenz.

Die einfache, ambulante Handhabung, die Verträglichkeit sowie die zugrunde liegende Wirkhypothese, welche auch die antiinflammatorische Komponente der N. trigeminus-Stimulation einbezieht, erwecken deshalb bei Forschern und Klinikern großes Interesse. Auch die beschriebenen Verbesserungsverläufe bei schwer erkrankten $\mathrm{Pa}$ tienten geben Hoffnung. Die Ergebnisse sind aber nur als vorläufige erste Befunde zu verstehen. Die kleine Fallzahl sowie die fehlende Kontroll- und Placebogruppe schränken die Interpretation der Daten deutlich ein.

Bedeutende Kritik muss an der ungenügenden und widersprüchlichen Beschreibung des Suizids geübt werden. Etwaige Zusammenhänge mit eTNS sind zur Folge nicht auszuschließen und bedürfen einer genaueren Untersuchung. Somit ist die eTNS bezüglich des Wirk-, Sicherheits- und Nebenwirkungsprofil als eine rein experimentelle Therapie einzustufen.
1. Cook IA, Abrams M, Leuchter AF. Trigeminal Nerve Stimulation for Comorbid Posttraumatic Stress Disorder and Major Depressive Disorder. Neuromodulation 2016, Jan 28 [epub ahead of print]

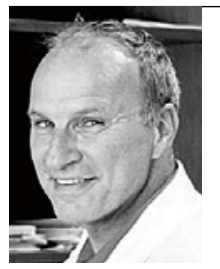

Prof. Dr. med. Andreas Conca Psychiatrischer Dienst im Gesundheitsbezirk Bozen, Italien

\title{
Transkranielle Gleichstromstimulation führt zu verminderter Aufmerksamkeit gegenüber bedrohlichen Stimuli
}

\begin{abstract}
Bei der transkraniellen Gleichstromstimulation soll ein schwacher Gleichstrom die Erregbarkeit kortikaler Regionen beeinflussen. Eine aktuelle Studie zeigt: Wenn die Elektroden über dem linken und rechten dorsolateralen präfrontalen Kortex positioniert werde, lässt die Aufmerksamkeit der Probanden gegenüber bedrohlichen Stimuli nach.
\end{abstract}

$B_{s}$ i der transkraniellen Gleichstromstimulation (transcrainial direct current stimulation, tDCS) handelt es sich um ein vergleichsweise junges Hirnstimulationsverfahren, das vor allem bei Depressionen Wirksamkeit zu zeigen scheint. Therapeutisches Grundprinzip ist die Applikation schwachen Gleichstroms mittels Oberflächenelektroden am wachen Patienten in subkonvulsiven Dosen, welche die Erregbarkeit kortikaler Regionen beeinflussen soll. Jedoch ist noch recht wenig über die optimale Wahl der Stimulationsparameter, etwa der

\begin{tabular}{|c|c|}
\hline 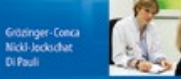 & Buchtipp \\
\hline $\begin{array}{l}\text { Elektrokonvulsions- } \\
\text { therapie kompakt }\end{array}$ & $\begin{array}{l}\text { Elektrokonvul- } \\
\text { sionstherapie } \\
\text { kompakt }\end{array}$ \\
\hline Derater & $\begin{array}{l}\text { Für Zuweiser und } \\
\text { Anwender }\end{array}$ \\
\hline \multicolumn{2}{|c|}{$\begin{array}{l}\text { M. Grözinger, A. Conca, T. Nickl-Jock- } \\
\text { schat, J. Di Pauli (Hrsg.) }\end{array}$} \\
\hline $\begin{array}{l}\text { Springer-Verla } \\
2013,240 \text { S., } 22 \\
\text { ISBN 978-3-64 }\end{array}$ & $\begin{array}{l}\text { Berlin, Heidelberg, } \\
\text { bb., Softcover, } 49,99 € \text {, } \\
25628-8\end{array}$ \\
\hline
\end{tabular}

Elektrodenplatzierung oder den genauen Wirkmechanismen, bekannt. Diesen Fragen widmete sich nun eine Studie, die mithilfe einer Testbatterie den Einfluss der tDCS auf die Emotionsverarbeitung an gesunden Probanden untersuchte [1]. Um den Einfluss der Elektrodenplatzierung abzubilden, wurde bei einem Teil der Probanden die Anode über dem linken, die Kathode über dem rechten dorsolateralen präfrontalen Kortex (DLPFC) positioniert. Bei einer weiteren Fraktion wurde die Anordnung dahingehend modifiziert, dass bei gleicher Position der Anode die Kathode rechts supraorbital angebracht wurde. Eine dritte Gruppe erhielt eine Scheinbehandlung.

Nach der Stimulation wurden verschiedene Verhaltenstests durchgeführt, die insbesondere bei Patienten mit Depression und Angststörungen Veränderungen feststellen. Dabei zeigte sich, dass die Aufmerksamkeit der Probanden gegenüber bedrohlichen Stimuli nachließ, wenn die Elektroden über linkem und rechtem DLPC positioniert waren. Bei den beiden anderen Gruppen konnte kein verändertes Verhalten festgestellt werden. Die Autoren verweisen darauf, dass ähnliche Veränderungen unter einer Therapie mit Anxiolytika zu beobachten seien. Dementsprechend könnte also der antidepressive Effekt der tDCS auf einer angstlösenden Wirkung beruhen. Zudem scheint die Elektrodenpositionierung einen deutlichen Einfluss auf die Wirksamkeit zu nehmen.

\section{Kommentar}

Die Studie weist mit $\mathrm{n}=20$ pro Gruppe eine relativ niedrige Probandenzahl auf. Zudem muss offen bleiben, inwieweit diese Ergebnisse tatsächlich eine therapeutische Relevanz haben, da die Untersuchungen nur an Gesunden durchgeführt wurden. Allerdings ergeben sich zwei wichtige Hinweise: Zum einen scheint die Wahl der Elektrodenplazierung relevant für den Outcome. Zum anderen könnte die tDCS vor allem über anxiolytische Effekte ihre antidepressive Wirkung entfalten. Zukünftige Studien werden sich diesen Fragen mit größeren Fallzahlen und dem Einschluss auch depressiver Patienten widmen müssen.

1. Ironside $\mathrm{M}$ et al. Frontal Cortex Stimulation Reduces Vigilance to Threat: Implications for the Treatment of Depression and Anxiety. Biol Psychiatry 2015, Jun 17. [epub ahead of print]

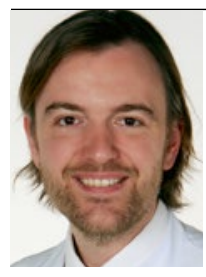

Prof. Dr. med. Thomas Nickl-Jockschat Klinik für Psychiatrie, Psychotherapie und Psychosomatik, RWTH Aachen 